J. Lake Sci. (湖泊科学) , 2015, 27(3): 519-526

http://www. jlakes. org. E-mail : jlakes@niglas.ac.cn

(C) 2015 by Journal of Lake Sciences

\title{
长江中下游浅水湖泊悬浮颗粒物吸收系数测定方法探讨
}

\author{
时志强 ${ }^{1,2}$, 张运林 $^{2 * *}$, 蔡同锋 ${ }^{1}$ \\ $(1$ : 江苏省环境监测中心, 南京 210036$)$ \\ ( 2 : 中国科学院南京地理与湖泊研究所湖泊与环境国家重点实验室, 南京 210008)
}

\begin{abstract}
摘 要: 对两种水体悬浮颗粒物吸收系数测定方法及相关计算进行对比研究. 通过长江中下游湖泊典型藻类的实 验室培养, 利用 T 方法和 T-R 方法分别对藻类颗粒物、藻类泥沙混合悬浊液进行吸收系数测定. 通过颗粒物光谱吸 收系数与叶绿素 a 之间的相关性关系, 对比了两种方法的测量稳定性. 通过对不同比例的藻类和无机悬浮颗粒物 (ISS) 的混合悬浊液进行分析, 获得了不同浊度水体悬浮物吸收光谱的变化情况. 结果表明, 在纯藻或者泥沙含量 较少的水体进行颗粒物吸收系数光谱测定时, T 方法和 T-R 方法均可以采用,并且均具有较高的测定精度. 然而, 在泥沙含量相对较高的浑浊水体,应尽量选取 T-R 方法进行颗粒物吸收光谱的测定, 以提高测定精度. 长江中下游 浅水湖泊由于底泥易受风浪影响发生再悬浮,因此在颗粒物吸收系数光谱测定中,当水体中 ISS 含量超过 $30 \mathrm{mg} / \mathrm{L}$ 时,应选择 T-R 方法.
\end{abstract}

关键词: 颗粒物吸收系数;悬浮颗粒物; T 方法;T-R 方法;浅水湖泊;长江中下游

\section{Determination of the spectral absorption coefficients of suspended particulate matters in the shallow lakes in the middle and lower reaches of Yangtze River}

\author{
SHI Zhiqiang ${ }^{1,2}$, ZHANG Yunlin ${ }^{2} \&$ CAI Tongfeng ${ }^{1}$ \\ (1: Environmental Monitoring Center of Jiangsu Province, Nanjing 210036, P. R. China) \\ (2: State Key Laboratory of Lake Science and Environment, Nanjing Institute of Geography and Limnology, Chinese Academy \\ of Sciences, Nanjing 210008, P. R. China)
}

\begin{abstract}
Two methods for measuring absorption coefficients of suspended particulate matters in water were studied and compared. Through the laboratory culturing of typical algae from the shallow lakes in the middle and lower reaches of Yangtze River, the algal particles and the suspension of algae and sediments were determined by Transmittance ( T) and Transmittance-Reflectance ( T-R) methods, respectively. The measuring stability of the two methods was compared according to the relationships between the spectral absorption coefficients and Chlorophyll-a of the suspended matters. To obtain absorption spectral changes of suspended matters at different levels of turbidity water, the suspension composed of algae and inorganic particles in different concentration gradients were analyzed. The results showed that both T and T-R methods could be applied and have high measurement precisions in pure algae or water containing less inorganic suspended particulate matters. However, the T-R method should be selected for the suspended particulate matter absorption spectra determination in turbid water. This can improve the measurement precision. Considering that the sediments in the study area are susceptible to wind and thus re-suspension, when the inorganic suspended particulate matters in water exceeded $30 \mathrm{mg} / \mathrm{L}$, the T-R method should be selected.
\end{abstract}

Keywords: Spectral absorption coefficients; suspended particulate matters; Transmittance method; Transmittance-Reflectance method; shallow lakes; middle and lower reaches of Yangtze River

* 江苏省杰出青年基金项目 (BK2012050)、中国科学院南京地理与湖泊研究所 “一三五”重点布局项目 ( NIGLAS2012135003) 和美国能源基金项目( G-1006-12776) 联合资助. 2014-04-24 收稿;2014-10-10 收修改 稿. 时志强(1985 ), 男,硕士;E-mail:szq19850518@ 126. com.

** 通信作者;E-mail:ylzhang@ niglas. ac. cn. 
悬浮颗粒物 (SS) 的光谱吸收是水体的固有光学特性, 是水色遥感的生物一光学模型、光辐射传输、初级 生产力估算和生态过程模拟的基本参数, 该参数的准确测量对于水体生物光学、水色遥感等诸多研究领域 有着重要意义 ${ }^{[1-2]}$. 从传统的无机有机组成来分, 悬浮颗粒物可以分为无机悬浮颗粒物 (ISS) 和有机悬浮颗

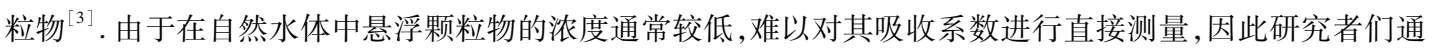
常采用定量化过滤技术将水体中的颗粒富集到滤膜上, 然后利用分光光度计进行其光吸收系数的测定, Mitchell 将该方法定为定量化过滤技术, 既 QFT 技术 ( Quantitative Filter Technique) ${ }^{[4]}$. QFT 技术根据具体操 作的不同可分为 Yentsch 在 1962 年创立的 T 方法 (Transmittance method) ${ }^{[5]}$ 和 Tassan 等在 1995 年建立的 TR 方法 (Transmittance-Reflectance method) ${ }^{[6]}$, 其中 T 方法是美国 NASA ( National Aeronautics and Space Administration) 发布的生物光学测量方法协议中建议的标准方法.

国内关于水体吸收系数的研究相对于国外起步较晚, 常用的两种颗粒物吸收系数测定方法均始于国 外,引人国内水体吸收系数研究后, 被研究者们广泛使用. 目前我国水体颗粒物吸收系数测定方法多采用 $\mathrm{T}$ 方法 ${ }^{[7-9]}$, 由于 T-R 方法测量和计算步骤较为复杂、对测量仪器要求较高, 在我国水体颗粒物吸收系数的测 量中运用较少 ${ }^{[10]}$. 由于水体理化性质的差异, 不同的实验分析方法的测量精度也存在一定差异 ${ }^{[11]}$. 在我国 水体光学研究中, 仅限于具体方法的使用, 关于两种颗粒物吸收系数测定方法在具体使用条件和测量精度 方面的研究较少.

由于长江中下游流域湖泊多为浅水湖泊, 易受风浪影响引起底泥的再悬浮, 从而导致悬浮物中泥沙含 量较高 ${ }^{[12-13]}$. 选择适合悬浮物较高水体的颗粒物吸收系数测定方法, 能够提高悬浮颗粒物吸收系数的精度, 更好地反映水体的固有光学特性. 本文根据国外学者提出的两种悬浮颗粒物吸收系数测定方法, 选择取自 长江中下游典型浅水湖泊 (太湖) 的底泥及长江中下游湖泊的优势藻种, 对两种测定方法在实验室控制条件 下的测定精度进行分析, 确定适合长江中下游浅水湖泊水体悬浮颗粒物的测定方法, 以期为长江中下游湖 泊悬浮颗粒物吸收系数的精确测定提供一定的理论依据.

\section{1 材料与方法}

\section{1 样品制备方法}

在实验室条件下培养长江中下游流域富营养化湖泊优势藻种铜绿微囊藻 (Microcystis aeruginosa) 和斜生 栅藻 (Scenedesmus obliquus), 取太湖底泥, 风干后进行研磨, 过 100 目的笁网. 在实验室取 $100 \mathrm{~L}$ 容器, 在混合 培养的藻中分别加入研磨的底泥, ISS 浓度梯度分别为 $5 、 15 、 30 、 45 、 60 、 90 、 250 、 500 \mathrm{mg} / \mathrm{L}$, 每次取 3 个平行 样,分别利用两种方法进行测定.

\section{2 两种方法测量及计算过程}

1.2.1 $\mathrm{T}$ 方法测量及计算 $\mathrm{T}$ 方法 ${ }^{[4,14-15]}$ 测定过程中, 颗粒的散射作用导致测量过程中的实际光程大于颗粒 样品在悬浮状态下的几何光程, 因此产生光程放大效应, 故由 QFT 方法得到的结果需要采用光程放大系数 $\beta$ 进行校正,计算公式为:

$$
\beta=\mathrm{OD}_{\mathrm{f}} / \mathrm{OD}_{\text {sus }}
$$

式中, $\mathrm{OD}_{\mathrm{f}}$ 为 $\mathrm{QFT}$ 方法所测得的滤膜上颗粒物的吸光度, $\mathrm{OD}_{\mathrm{sus}}$ 为悬浮液中颗粒的吸光度.

对 QFT 所得结果进行光程放大系数校正后, 即将 $\mathrm{OD}_{\mathrm{f}}$ 值转换成 $\mathrm{OD}_{\text {sus }}$ 值, 颗粒体积吸收系数 $a$ 即可通过 式(2)计算求得:

$$
a=2.3030 \mathrm{OD}_{\text {sus }} \cdot S / V
$$

式中, $S$ 为颗粒在滤膜上所占的实际面积, $V$ 为过滤水样的体积.

在采用 $\mathrm{T}$ 方法进行光程放大系数的研究过程中, 一些研究者假定 $\beta$ 值为常数, 即 $\beta$ 值不随 $\mathrm{OD}_{\mathrm{f}}$ 、波长变

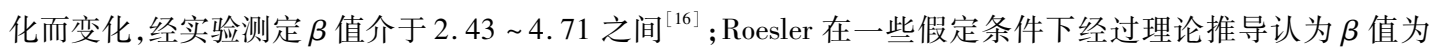
$2^{[17]}$; 但更多研究者发现 $\beta$ 值并不恒定, Sozik 等 ${ }^{[18]}$ 通过对 Mitchell 等 $^{[19]}$ 的数据进行分析后, 认为 $\mathrm{OD}_{\text {sus }}$ 与 $\mathrm{OD}_{\mathrm{f}}$ 存在着较好的幂函数关系.

本研究悬浮颗粒物的吸收采用 QFT 测定, 用直径 $25 \mathrm{~mm}$ 的 GF/F (Whatman 公司) 滤膜过滤水样 (根据 
水里悬浮物浓度决定过滤的体积), 在 UV-2250 型分光光度计下测定滤膜上悬浮颗粒物的吸光度, 用同样湿 润程度的空白滤膜做参比, 为减少散射误差, 被测滤膜尽量靠近分光光度计的接受窗口, 在 $350 \sim 800 \mathrm{~nm}$ 间 每隔 $1 \mathrm{~nm}$ 测定一次吸光度,用各波段的吸光度减去 $750 \mathrm{~nm}$ 波长的吸光度, 采用 Cleveland 等 $^{[14]}$ 提出的公式 进行放大因子校正:

$$
\mathrm{OD}_{\mathrm{s}}=0.3780 \mathrm{D}_{\mathrm{f}}+0.5230 D_{\mathrm{f}}^{2} \quad \mathrm{OD}_{\mathrm{f}} \leqslant 0.4
$$

式中, $\mathrm{OD}_{\mathrm{s}}$ 为校正后的滤膜上悬浮颗粒物吸光度.

滤膜上悬浮颗粒物的光谱吸收系数计算公式为:

$$
a_{\mathrm{p}}(\lambda)=2.3030 \mathrm{D}_{\mathrm{s}}(\lambda) \cdot S / V
$$

式中, $V$ 为被过滤水样的体积.

1.2.2 T-R 方法测量及计算 T-R 法测定样品的光学密度采用以下公式计算:

$$
\mathrm{OD}_{\mathrm{fp}-\mathrm{TR}}(\lambda)=-\lg \left(1-\frac{1-\rho_{\mathrm{T}}+R_{\mathrm{f}}\left(\rho_{\mathrm{T}}-\rho_{\mathrm{R}}\right)}{1+R_{\mathrm{f}} \cdot \rho_{\mathrm{T}} \cdot \tau}\right)
$$

式中, $\rho_{\mathrm{T}}$ 为透射测量模式下测量得到的通过样品与参比的光谱通量的比值, $\rho_{\mathrm{R}}$ 为反射测量模式下测得 的样品与参比的光谱通量的比值, $R_{\mathrm{f}}$ 为空白 $\mathrm{GF} / \mathrm{F}$ 滤纸的反射比, $\tau$ 为 $1^{[20]}, \mathrm{OD}_{\mathrm{fp}-T \mathrm{~T}}(\lambda)$ 为样品滤纸的吸 光度.

$$
\begin{gathered}
\beta=\left[0.423+0.4790 D_{\mathrm{fp}-\mathrm{TR}}(\lambda)\right]^{-1} \\
a_{\mathrm{p}}(\lambda)=\frac{2.303 S}{\beta \cdot V_{\mathrm{f}}} \mathrm{OD}_{\mathrm{fp}-\mathrm{TR}}(\lambda)
\end{gathered}
$$

式中, $a_{\mathrm{p}}(\lambda)$ 为悬浮颗粒物吸收系数, $\mathrm{OD}_{\mathrm{fp}}(\lambda)$ 为 $\mathrm{T}-\mathrm{R}$ 法测量获得的样品滤纸的吸光度, $S$ 为根据滤纸上富集 有悬浮颗粒物部分的滤纸直径 $\left(D_{\mathrm{r}}\right)$ 计算得到的净面积 ${ }^{[20]}$.

\section{2 结果与讨论}

\section{1 两种方法在纯藻测定中的对比研究}

对培养的两类藻利用两种方法进行光谱测定, 得到其悬浮颗粒物吸收系数, 利用有效光合辐射 (PAR) 波段 (400 700 nm) 吸收系数的平均值与叶绿素 a(Chl. a) 浓度进行相关性分析. 从图 1 还可以发现,利用 T 方法和 T-R 方法分别测定同一组藻时, T-R 方法所测得 PAR 波段的平均吸收系数与 Chl. a 的相关性系数略 大于 $\mathrm{T}$ 方法的测定结果, 说明 T-R 方法的测定结果更接近真实值. 对 T-R 方法和 T 方法在有效光合辐射波 段的吸收系数进行标准偏差分析, 结果分别为: 铜绿微囊藻: 3.097、3. 227, 斜生栅藻: 1.259、1.400, 说明 T-R 方法在纯藻测定中的稳定性要略高于 T 方法.

分析其悬浮颗粒物吸收系数光谱 (图 2) 发现,随着藻类浓度的增加,各波段吸收系数均明显增加,铜绿 微囊藻和斜生栅藻吸收光谱在谱型上较为相似, 在 $440 、 675 \mathrm{~nm}$ 叶绿素特征吸收波长处, 存在两个明显的特 征吸收峰. 两类藻在 $450 、 625 \mathrm{~nm}$ 处存在微弱的差异,这可能是藻蓝素的吸收引起的.

利用 T 方法和 T-R 方法, 分别对铜绿微囊藻和斜生栅藻进行光谱测定, 对测定结果进行分析得到表 1. 两种方法所测得的悬浮颗粒物吸收系数有效光合辐射波段 ( $400 \sim 700 \mathrm{~nm})$ 的平均结果差别不大, 平 均差异分别为 $4.34 \%$ (铜绿微囊藻) 和 $4.92 \%$ (斜生栅藻), $\mathrm{T}$ 方法略高于 T-R 方法. 由于 T 方法无法 消除滤膜上颗粒后向散射及颗粒与滤膜之间多次反射而导致测定过程中对颗粒吸收的高估, 从而引 起颗粒物吸收系数的放大. T 方法在测定后进行计算时, 根据纯藻在 $750 \mathrm{~nm}$ 处的吸收系数接近 0 的特 点,通常在 $750 \mathrm{~nm}$ 处进行校正. 而 T-R 方法则是利用积分球,经过一次透射和一次反射测定消除了后 向散射的影响,通过相关校正计算后测定结果与真实的吸收系数值也比较接近. 对比两种方法在测定 两种藻类的测量结果差异时发现,两者存在一定差异, 但差异不大,因此两种方法在测定纯藻颗粒物 吸收系数时均可以使用. 

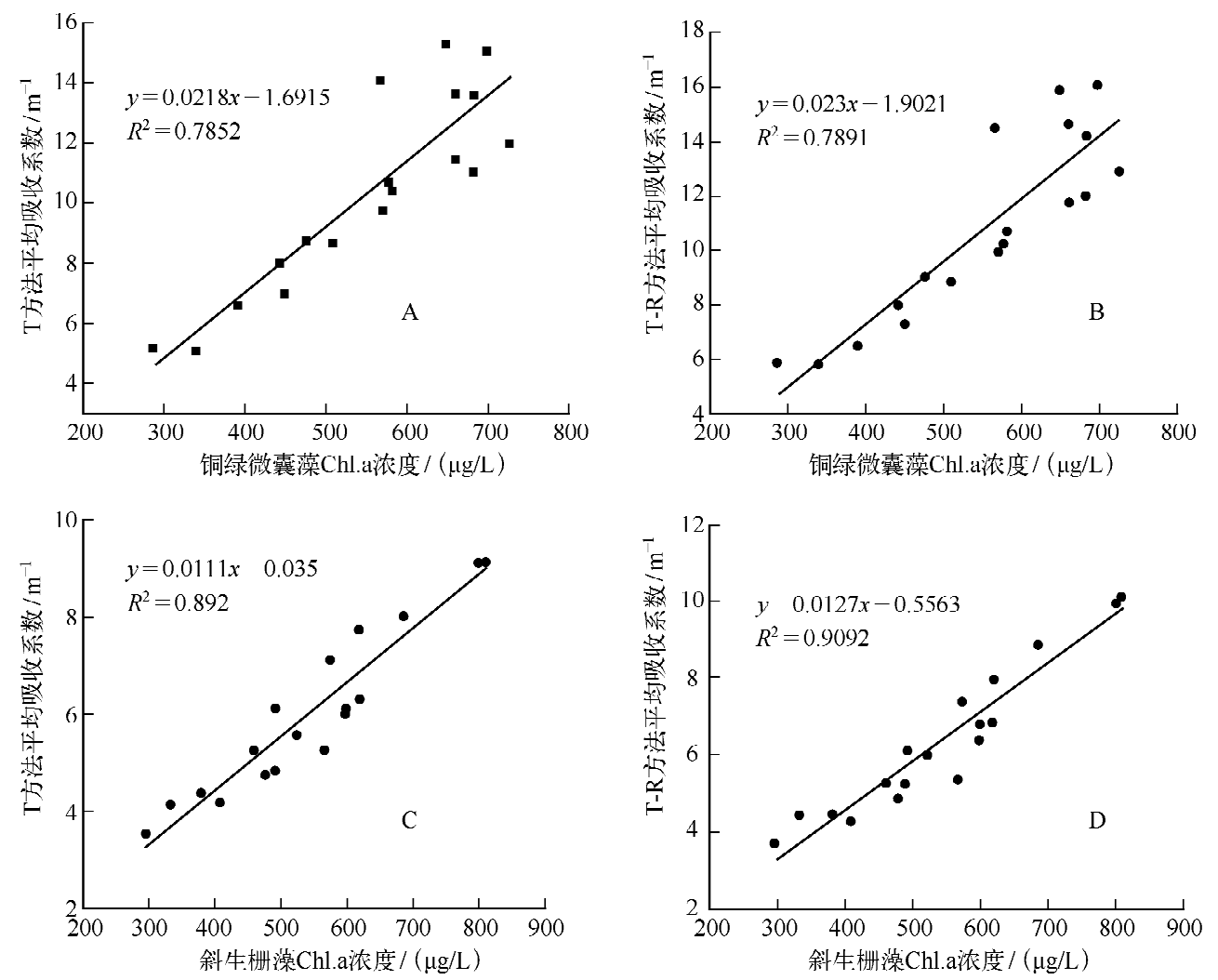

图 $1 \mathrm{Chl}$. a 浓度与光谱(有效光合辐射波段) 吸收系数相关性分析

Fig. 1 Correlations between PAR absorption coefficients and Chl. a concentration
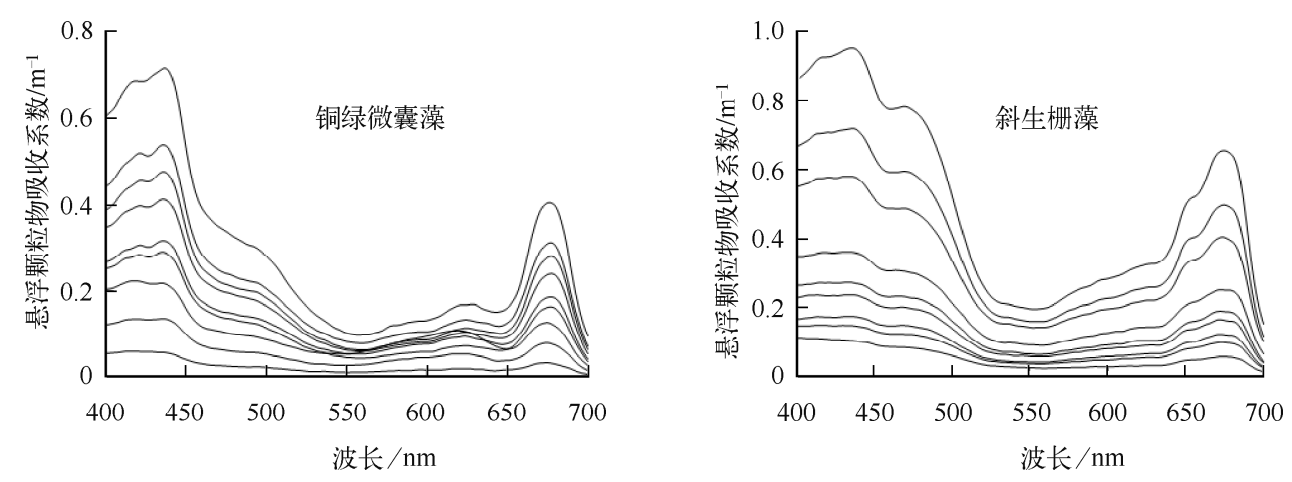

图 2 铜绿微囊藻和斜生栅藻的吸收系数光谱

Fig. 2 The spectral absorptions of Microcystis aeruginosa and Scenedesmus obliquus 
表 1 两种方法测定纯藻吸收系数的差异 *

Tab. 1 The difference of the two methods for measuring absorption coefficient

\begin{tabular}{crrrrrrrrr}
\hline 铜绿微囊藻 & $\mathrm{T}-\mathrm{R}$ & $\mathrm{T}$ & 差异/\% & $\mathrm{B}-\mathrm{Chl} . \mathrm{a} /(\mu \mathrm{g} / \mathrm{L})$ & 斜生栅藻 & $\mathrm{T}-\mathrm{R}$ & $\mathrm{T}$ & 差异/\% & $\mathrm{G}-\mathrm{Chl} . \mathrm{a} /(\mu \mathrm{g} / \mathrm{L})$ \\
\hline B-1 & 5.18 & 5.87 & 13.32 & 286.6 & $\mathrm{G}-1$ & 3.54 & 3.7 & 4.24 & 295.6 \\
B-2 & 5.10 & 5.83 & 14.31 & 338.9 & $\mathrm{G}-2$ & 4.14 & 4.4 & 7.00 & 333.9 \\
B-3 & 6.62 & 6.49 & -1.96 & 390.4 & $\mathrm{G}-3$ & 4.36 & 4.4 & 1.61 & 380.3 \\
B-4 & 6.98 & 7.27 & 4.15 & 449.3 & $\mathrm{G}-4$ & 4.17 & 4.3 & 2.88 & 408.1 \\
B-5 & 8.01 & 7.98 & -0.37 & 442.0 & $\mathrm{G}-5$ & 5.24 & 5.3 & 0.57 & 460.4 \\
B-6 & 8.74 & 9.06 & 3.66 & 476.5 & $\mathrm{G}-6$ & 4.75 & 4.9 & 2.32 & 477.9 \\
B-7 & 8.66 & 8.84 & 2.08 & 509.2 & $\mathrm{G}-7$ & 4.83 & 5.3 & 8.70 & 489.8 \\
B-8 & 9.72 & 9.97 & 2.57 & 570.0 & $\mathrm{G}-8$ & 5.60 & 6.0 & 6.96 & 524.0 \\
B-9 & 10.41 & 10.71 & 2.88 & 581.8 & $\mathrm{G}-9$ & 6.11 & 6.1 & 0 & 492.9 \\
B-10 & 10.70 & 10.24 & -4.30 & 576.8 & $\mathrm{G}-10$ & 5.26 & 5.4 & 2.28 & 567.0 \\
B-11 & 11.43 & 11.77 & 2.97 & 660.7 & $\mathrm{G}-11$ & 6.00 & 6.4 & 5.83 & 597.4 \\
B-12 & 11.03 & 12.03 & 9.07 & 682.1 & $\mathrm{G}-12$ & 6.30 & 6.8 & 8.25 & 618.2 \\
B-13 & 12.00 & 12.90 & 7.75 & 726.0 & $\mathrm{G}-13$ & 6.10 & 6.8 & 11.15 & 599.0 \\
B-14 & 14.10 & 14.48 & 2.70 & 567.2 & $\mathrm{G}-14$ & 7.11 & 7.4 & 3.66 & 574.0 \\
B-15 & 15.27 & 15.87 & 3.93 & 648.9 & $\mathrm{G}-15$ & 7.73 & 8.0 & 2.98 & 619.0 \\
B-16 & 15.10 & 16.10 & 6.69 & 699.0 & $\mathrm{G}-16$ & 8.02 & 8.8 & 10.22 & 686.0 \\
平均 & 9.94 & 10.34 & 4.34 & 537.8 & 平均 & 5.58 & 5.9 & 4.92 & 507.7 \\
\hline
\end{tabular}

* “B” 表示铜绿微囊藻; “G” 表示斜生栅藻; 差异表示 “ T”方法测定结果与 “ T-R” 方法测定结果的差值和“ T”方法测定结 果的比值; B-Chl. a、G-Chl. a 分别表示铜绿微囊藻和斜生栅藻的叶绿素 a 浓度.

\section{2 两种方法在测定含泥沙悬浮颗粒物时的结果对比}

由于两种藻类在单独测量时吸收系数谱线差异不大, 为更好地模拟自然状况, 取实验配制藻类和太湖 沉积物混合水样, 每次取 3 个平行样. 将所取样品通过两种悬浮颗粒物测量方法分别进行测定. 对比混合藻 光谱和加人底泥的测量结果可知, 在加人底泥量较少时仍具有混合藻光谱类似的特征, 在 $440 、 675 \mathrm{~nm}$ 处有 两个明显的叶绿素吸收峰. 随着泥沙量不断加大, 混合泥沙样品与纯样品出现了明显的差异, $440 \mathrm{~nm}$ 处叶绿 素的特征吸收峰逐渐消失, 这主要是因为泥沙悬浮颗粒物样品吸收光谱呈指数衰减, 随着样品泥沙含量的 不断增加, 在短波部分吸收系数不断增加, 440 nm 处原有的叶绿素吸收峰被泥沙光谱的吸收系数所掩盖. 由 光谱图也能发现, 随着泥沙量的不断增加,在短波部分吸收系数增长得非常快,而在长波部分增长得相对较 慢,因此在 $675 \mathrm{~nm}$ 处仍具有一个明显的叶绿素吸收峰(图 3 ).

对比分析不同浓度梯度的无机悬浮颗粒物水样, 发现当无机悬浮颗粒物含量为 $5 \mathrm{mg} / \mathrm{L}$ 时, 两种测定方 法的差异不大,在整个 PAR 波段 T 方法略高于 T-R 方法, 当无机悬浮颗粒物含量为 $30 \mathrm{mg} / \mathrm{L}$ 时,两种方法的 测定结果已经出现明显差异 (图 4). 随着无机悬浮颗粒物浓度的增加, 在各波长处吸收系数的差异均有所增 大. 分析原因, 主要是因为 $\mathrm{T}$ 方法认为在 $750 \mathrm{~nm}$ 处浮游藻类的吸收系数几乎为 0 , 因此在 $750 \mathrm{~nm}$ 处做相应 的校正,而 T-R 方法未在 $750 \mathrm{~nm}$ 处进行归 0 校正. 相关研究表明 ${ }^{[21-22]}$, 无机悬浮颗粒物在 $750 \mathrm{~nm}$ 处仍具有 一定的吸收系数, 并且随着无机悬浮颗粒物浓度的增大而增大, 由 T-R 方法的测量结果 (图 4 ) 也能看出. 虽 然散射会增大 $\mathrm{T}$ 方法的研究结果, 但直接利用 $750 \mathrm{~nm}$ 处吸收系数为 0 进行校正, 在无机悬浮颗粒物存在时, 由于将 $750 \mathrm{~nm}$ 处无机悬浮颗粒物的吸收系数也视为 0 , 会低估其吸收系数,特别是当无机悬浮颗粒物浓度 较高时由于忽略无机悬浮颗粒物在 $750 \mathrm{~nm}$ 处的吸收引起的吸收系数,低估将远高于散射引起的吸收系数升 高. 因此,通过对比可以发现, 当所测量目标的组成成分发生变化时,两种测量方法校正因子的差别是影响 测量结果的主要因素, 特别是 $\mathrm{T}$ 方法在 $750 \mathrm{~nm}$ 处的直接归 0 校正, 由于忽略了无机悬浮颗粒物在长波段的 吸收系数特点,会对测量结果产生较大影响.

由表 2 可知,通过对藻类混合泥沙后吸收系数的测定结果在 $400 \sim 700 \mathrm{~nm}$ 处积分对比,在加人泥沙量相 对较少的时候, 两种方法测量的结果差异不大,随着泥沙的不断加人, 两种方法测定结果的差异由 $-0.76 \%$ 

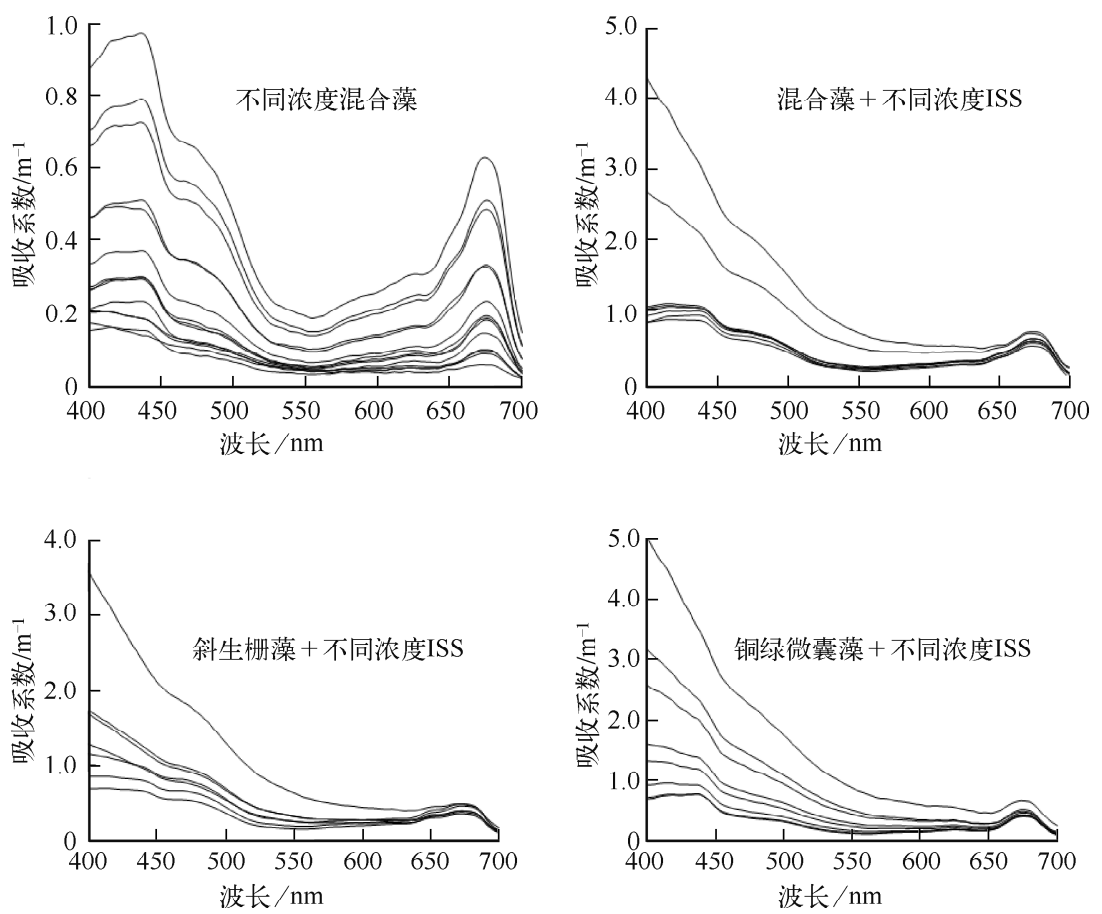

图 3 藻类混合无机悬浮颗粒物后的光谱变化

Fig. 3 Changes in spectra when the algae was mixed with inorganic suspended particles
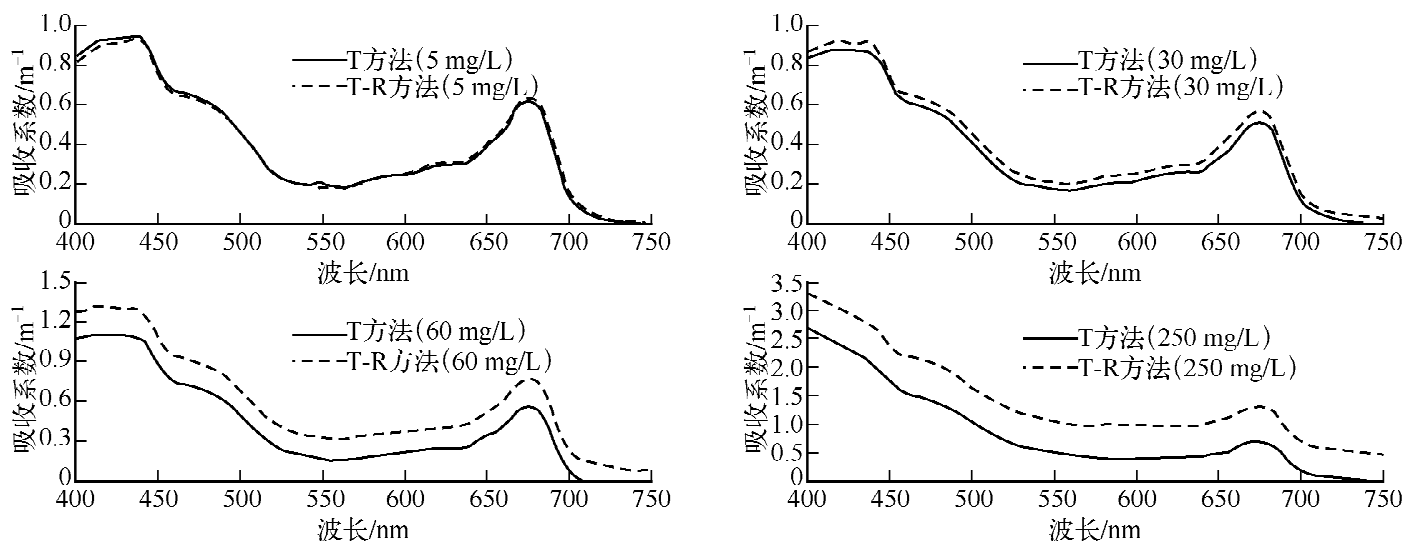

图 4 两种方法测定混人不同浓度无机悬浮颗粒物水样光谱对比

Fig. 4 Changes in spectra when suspension by algae and inorganic particles in different concentrations

增大到 $38.95 \%$, 并且 T-R 方法的测定结果在无机悬浮颗粒物含量较大时, 明显高于 T 方法的测定结果. 上 文中已阐述这主要是因为悬浮泥沙样品或者含悬浮泥沙的混合液在 $750 \mathrm{~nm}$ 附近对光仍有一定的吸收作用, 且随着无机悬浮颗粒物浓度的增加而增大, 而 $\mathrm{T}$ 方法利用浮游植物在 $750 \mathrm{~nm}$ 附近波段吸收系数为 0 的 $\mathrm{OD}_{\text {sus }}$ 假设, 在计算时进行光谱数据的校正, 因此在泥沙含量过高时, 由于忽略了无机悬浮颗粒物在 $750 \mathrm{~nm}$ 处的吸收, 在进行校正时, 有效光合辐射波段各波长处的吸收系数将减小, 且减小的数值将高于短波高散射 引起的增大, 而 T-R 方法不需要在 $750 \mathrm{~nm}$ 处进行校正, 所以测定结果高于 $\mathrm{T}$ 方法的测定结果, 且更接近于真 实值. 
表 2 不同无机悬浮颗粒物浓度下两种方法测定结果在 $400 \sim 700 \mathrm{~nm}$ 波段积分值之间的差异 *

Tab. 2 The difference of the results in the $400-700 \mathrm{~nm}$ waveband integral value by the two methods under different inorganic particle concentrations

\begin{tabular}{|c|c|c|c|c|c|c|c|c|}
\hline & $\begin{array}{c}\text { 混合藻 } \\
+ \text { ISS1 }\end{array}$ & $\begin{array}{r}\text { 混合藻 } \\
+ \text { ISSS2 }\end{array}$ & $\begin{array}{r}\text { 混合藻 } \\
+ \text { ISS3 }\end{array}$ & $\begin{array}{r}\text { 混合藻 } \\
+ \text { ISS4 }\end{array}$ & $\begin{array}{r}\text { 混合藻 } \\
+ \text { ISS5 }\end{array}$ & $\begin{array}{r}\text { 混合藻 } \\
+ \text { ISS6 }\end{array}$ & $\begin{array}{c}\text { 混合藻 } \\
+ \text { ISS7 }\end{array}$ & 平均 \\
\hline ISS 浓度/( $\mathrm{mg} / \mathrm{L})$ & 5 & 15 & 30 & 45 & 60 & 250 & 500 & 129.28 \\
\hline $\mathrm{T}$ 法 & 0.46 & 0.49 & 0.51 & 0.53 & 0.57 & 0.96 & 1.36 & 0.70 \\
\hline T-R 法 & 0.46 & 0.49 & 0.56 & 0.61 & 0.66 & 1.56 & 2.23 & 0.94 \\
\hline 差异/\% & -0.76 & -1.05 & 8.85 & 14.80 & 16.32 & 38.25 & 38.95 & 16.48 \\
\hline
\end{tabular}

对不同浓度梯度无机悬浮颗粒物两种测定方法之间的差异进行分析,随着无机悬浮颗粒物浓度的不断 增大, 两种方法测定的吸收系数结果的差异也在不断增加, 当无机悬浮颗粒物浓度达到 $30 \mathrm{mg} / \mathrm{L}$ 时, 两种测 定方法之间的差异达到 $8.85 \%$. 由于 T-R 方法是在积分球中进行的测定,结果受到散射的影响较小, 不用进 行散射校正,避免了因校正而引起的误差. 因此在对无机悬浮颗粒物含量较高的水样进行吸收系数测定中, 当无机悬浮颗粒物含量超过 $30 \mathrm{mg} / \mathrm{L}$ 时,两种方法的测定结果已经产生较大差异,应尽量选用 T-R 方法进行 测定, 从而提高吸收系数的测定精度.

\section{3 结论}

1 ) 通过对比不同浓度藻类颗粒物吸收系数与叶绿素之间的关系可知, T-R 方法的测量稳定性要好于 T 方法.

2 ) 通过对比两种悬浮颗粒物吸收系数的测定方法,在纯藻或者泥沙含量较少的水体进行悬浮颗粒物吸 收系数光谱测定时两种测定方法均可以采用,并且均具有较高的测定精度.

3 ) 在泥沙含量相对较高的长江中下游浅水湖泊,应尽量选取 T-R 方法进行悬浮颗粒物吸收光谱的测 定,以提高测定精度.

\section{4 参考文献}

[ 1 ] 张运林,秦伯强,陈伟民等. 太湖水体中悬浮物研究. 长江流域资源与环境,2004,13(3):266-271.

[ 2 ] 张运林. 湖泊光学研究进展及其展望. 湖泊科学,2011,23(4) :483-497.

[ 3 ] Kirk JTO. Light and photosynthesis in aquatic ecosystem. Cambridge: Cambridge University Press, 1994.

[ 4 ] Mitchell BG. Algorithms for determining the absorption coefficient of aquatic particulates using the quantitative filter technique( QFT). Proceeding of the International Society for Optical Engineering, 1990,1302:137-148.

[ 5 ] Yenrsch CS. Measurement of visible light absorption by particulate matter in the ocean. Limnology and Oceanography, $1962,7: 207-217$.

[ 6 ] Tassan S, Ferrari GM. An alternative approach to absorbtion measurement of aquatic particls retained on filters. Limnology and Oceanography, $1995, \mathbf{4 0}: 1358-1368$.

[ 7 ] 王远东,刘殿伟,宋开山等. 吉林省查干湖水体悬浮颗粒物光谱吸收特性研究. 光谱学与光谱分析, 2011,31(1): 162-167.

[ 8 ] Gai LY, Liu ZJ, Zhang JX. Absorption coefficient characteristics of the Three Gorges Dam water. Journal of Remote Sensing. $2010, \mathbf{1 4}(2): 313-332$.

[ 9 ] 王桂芬, 曹文熙, 杨顶田等. 基于光谱相关关系的海水总悬浮颗粒物吸收光谱的分解. 光谱学与光谱分析,2009,29 ( 1 ) :201-206.

[10］余小龙,沈 芳, 张晋芳. 影响悬浮颗粒物吸收系数测量的相关因素研究. 环境科学, 2013,34(5):1745-1752.

[11] 朱建华, 周虹丽, 李铜基. 基于小球藻的 T-R 方法光程放大校正因子的适用性研究. 海洋技术,2010,29(1):40-45.

[12] 时志强,张运林,刘明亮等. 湖北东湖、梁子湖和洪湖颗粒物吸收光谱特征分析. 长江流域资源与环境, 2011,20 (6) :736-743.

[13] 施 坤, 李云梅, 吕 恒等. 太湖水体颗粒物散射的剖面分布及其影响因素分析. 环境科学,2010,31(3):598-605. 
[14] Cleveland JS, Weidemann AD. Quantifying absorption by aquatic particles: a multiple scattering correction for glass-fiber filter. Limnology and Oceanography, $1993,38(6)$ :1321-1327.

[15] Ferrari GM, Tassan S. A method using chemical oxidation to remove light absorption by phytoplankton pigments. Journal of Phycology, 1999,35(5):1090-1098.

[16] Kishino M, Takahashi M, Okami N et al. Estimation of the spectral absorption coefficients of phytoplankton in the sea. Bulletin of Marine Science, 1985,37 (2) :634-642.

[17] Roesler CS. Theoretical and experimental approaches to improve the accuracy of particulate absorption coefficients derived from the quantitative filter technique. Limnology and Oceanography, $1998, \mathbf{4 3}(7): 1649-1660$.

[18] Sozik HM, Mitchell BG. Light absorption by phytoplankton, photo-synthetic pigments and detritus in the California Current System. Deep Sea Research Part I, $1995, \mathbf{4 2}$ (10):1717-1748.

[19] Mitchell BG, Kiefer DA. Chlorophyll a specific absorption and fluorescence excitation spect ra for light limited phytoplankton. Deep Sea Research Part I, $1988,35: 639-663$.

[20] Tassan S, Ferrari GM. A sensitivity analysis of the "Transmittance-Reflectance" method for meaning light absorption by aquatic particles. Journal of Plankton Research, $2002,24(8)$ : 757-774.

[21] 孙德勇,李云梅, 王 桥等. 太湖水体中悬浮颗粒物的比吸收光谱特性及其参数化分析. 环境科学学报, 2009,29 (9) : 1820-1829.

[22] 朱建华,李 铜. 基黄东海非色素颗粒与黄色物质的吸收系数光谱模型研究. 海洋技术,2004,23(2):7-13. 